\title{
Preface-JES Focus Issue on 4D Materials and Systems
}

\section{Rangachary Mukundan, ${ }^{1, *}$ Hidemitsu Furukawa, ${ }^{2, *}$ Giuseppe Milano, ${ }^{3, *}$ Hiroyuki Matsui, ${ }^{4, *}$ Tsukasa Yoshida, ${ }^{5, *}$ Sathish K. Sukumaran, ${ }^{3,6}$ Jessica Koehne, ${ }^{7, *}$ Peter Hesketh, Kafil M. Razeeb, ${ }^{,}{ }^{*}$ Luca Magagnin, ${ }^{10, *}$ Ajit Khosla, ${ }^{2, *, z}$ and Johan Moulin ${ }^{11, *}$}

${ }^{1}$ Los Alamos National Laboratory, Los Alamos, New Mexico 87545, USA

${ }^{2}$ Department of Mechanical Systems Engineering, Graduate School of Science and Engineering, Yamagata University, Yonezawa, Yamagata 992-8510, Japan

${ }^{3}$ Department of Organic Material Science, Yamagata University, Yonezawa 992-8510, Japan

${ }^{4}$ Yamagata University, Yonezawa 992-8510, Japan

${ }^{5}$ Research Center for Organic Electronics (ROEL), Yamagata University, Yonezawa, Yamagata, 992-8510, Japan

${ }^{6}$ Department of Polymeric and Organic Materials Engineering, Yamagata University, Yonezawa, Japan

${ }^{7}$ NASA, Moffett Field, California 94035, USA

${ }^{8}$ Georgia Tech Research Institutes, Food Processing Center, Atlanta, Georgia 30318, USA

${ }^{9}$ Tyndall National Institute, University College Cork, Lee Maltings, Cork T12 R5CP, Ireland

${ }^{10}$ Department of Chemistry, Materials and Chemical Engineering "Giulio Natta", Politecnico di Milano, 20131

Milan, Italy

${ }^{11}$ Center for Nanoscience and Nanotechnology (C2N), CNRS, Univ. Paris-Sud, Université Paris-Saclay, 91405 Orsay cedex, France

(C) 2019 The Electrochemical Society. [DOI: 10.1149/2.0481909jes] Published June 11, 2019. This paper is part of the JES Focus Issue on 4 D Materials and Systems.

This focus issue of the Journal of The Electrochemical Society (JES) is devoted the First International Conference on 4D Materials and Systems, Yamagata University, Japan and Microfluidics, MEMS/NEMS Sensors and Devices II symposium, held at the Seattle, Washington, Meeting of the Society, May 13-17, 2018.

It has been an exciting opportunity to collect together selected papers from authors who were invited to present talks at 4DMS from August 26-30, 2018, at the Faculty of Engineering at Yamagata University, and at Microfluidics, MEMS/NEMS Sensors and Devices II symposium. The 4DMS conference had five parallel tracks: (1) Gel Symposium, (2) Flexible and Printed Electronics, (3) Material Processing, (4) Electrochemical Materials and Devices for Energy Conversion and Storage, and (5) Sensors and Systems.

We would like to highlight a few articles that reflect emerging trends in the field of 4D materials, Microfluidics, MEMS/NEMS Sensors and Devices. A Perspective article by Muthukumaran
Packirisamy et al., on Bio-Nano-Interaction in Treatment and Management of Cancer; development of ABE-Stat, a Fully Open-Source and Versatile Wireless Potentiostat Project Including Electrochemical Impedance Spectroscopy by Daniel M. Jenkins et al.; 4D Printing of Smart Stimuli-Responsive Polymers by Raffaella Suriano et al., Editors' Choice articles on Artificial Intelligence Based Mobile Application for Water Quality Monitoring by Sushanta Mitara et al., and Development of Screen-Printed Flexible Multi-Level Microfluidic Devices with Integrated Conductive Nanocomposite Polymer Electrodes on Textiles by Bonnie Gray et al.

The guest editors would like to thank all of the authors who have contributed manuscripts for this issue, all of the reviewers, JES Editor Robert Savinell for his guidance, and importantly the ECS staff, especially Beth Schademann, Paul Cooper, Annie Goedkoop, Beth Fisher Craanen, and Andrea Guenzel for making this issue a success. 